Cite this: Analyst, 2013, 138, 3221

\title{
Integrated label-free silicon nanowire sensor arrays for (bio)chemical analysist
}

\author{
Arpita De, Jan van Nieuwkasteele, Edwin T. Carlen* and Albert van den Berg
}

\begin{abstract}
We present a label-free (bio)chemical analysis platform that uses all-electrical silicon nanowire sensor arrays integrated with a small volume microfluidic flow-cell for real-time (bio)chemical analysis and detection. The integrated sensing platform contains an automated multi-sample injection system that eliminates erroneous sensor responses from sample switching due to flow rate fluctuations and provides precise sample volumes down to $10 \mathrm{nl}$. Biochemical sensing is demonstrated with real-time 15-mer DNA-PNA (peptide nucleic acid) duplex hybridization measurements from different sample concentrations in a low ionic strength, and the equilibrium dissociation constant $K_{\mathrm{D}} \approx 140 \mathrm{nM}$ has been extracted from the experimental data using the first order Langmuir binding model. Chemical sensing is demonstrated with $\mathrm{pH}$ measurements from different injected samples in flow that have sensitivities consistent with the gate-oxide materials. A differential sensor measurement configuration results in a $30 \times$ reduction in sensor drift. The integrated label-free analysis platform is suitable for a wide range of small volume chemical and biochemical analyses.
\end{abstract}

Received 31st October 2012 Accepted 28th March 2013

DOI: $10.1039 / \mathrm{c} 3 a n 36586 \mathrm{~g}$

www.rsc.org/analyst
Isothermal titration calorimetry and differential scanning calorimetry are truly label-free methods that can measure the thermodynamic binding properties in the native biomolecular environment. ${ }^{3}$ Although the calorimetric methods are extremely powerful, they are currently limited to large sample volumes and concentrations. Alternatively, the receptor molecule can be tethered directly to a sensor surface that can measure in realtime the change of surface properties as the target analyte binds to the receptors. Currently, many different surface-based labelfree sensor systems are available that use different detection phenomena, such as surface plasmon resonance, optical interferometry, optical gratings, quartz crystal microbalance, and surface acoustic waves. ${ }^{4}$ Surface plasmon resonance sensors $^{5}$ and surface plasmon resonance imaging sensors ${ }^{6}$ are well-established label-free techniques, but they require sophisticated optical instrumentation for signal readout and have limited sensitivity, especially for small molecular weight molecules. All-electrical sensor configurations are promising, such as the direct readout of biomolecular binding using electrochemical labels, ${ }^{7}$ or semiconductor transistors that measure the intrinsic electronic biomolecular charge on the gate-oxide using a field-effect to modulate the device conductance. ${ }^{8,9}$

There has been a steady increase in reports on ultrasensitive field-effect biosensors over the past several years based on semiconductor nanowires. ${ }^{10}$ One-dimensional electrical semiconductor nanowire sensors are particularly important due to their potential for large-scale, high-density integration and interfacing to conventional electronic systems, and hence are attractive for low cost portable sensing systems. Silicon nanowire (Si-NW) field-effect transistor (FET) biosensors have been
BIOS/Lab on a Chip Group, MESA+ Institute for Nanotechnology, University of Twente, Postbus 217, 7500 AE Enschede, The Netherlands.E-mail: e.t.carlen@utwente.nl; Fax: +31 (0)53 489 3595; Tel: +31 (0)534895399

† Electronic supplementary information (ESI) available: Multi-sample injection, hydraulic network design, and measured Si-NW sensor responses to sample flow rate changes. See DOI: 10.1039/c3an36586g 
reported extensively for highly sensitive, label-free, and realtime detection of biomolecular binding of DNA and proteins. ${ }^{\mathbf{1 1 , 1 2}}$ The high detection sensitivity of Si-NW biosensors has been attributed to their large surface-to-volume ratio and the three-dimensional multi-gate structure; both contribute to the improved sensitivity compared to conventional planar devices. However, device and technology developments have been limited to a small number of research laboratories typically relying on expensive nanolithography, specialized equipment and processes, or difficulties associated with alignment and electrical contacting into large sensor arrays. We recently reported a new scalable Si-NW sensor technology based on conventional microfabrication that does not require expensive nanolithography and can be implemented into large sensing arrays. ${ }^{\mathbf{1 3}}$ The main advantage of our technology is that dense SiNW sensor arrays, with precisely controlled dimensions and atomically smooth surfaces, can be manufactured in any conventional clean room, and can be integrated with small volume microfluidic channels with conventional bonding and clamping techniques.

In this article, we present an integrated label-free analysis platform that is suitable for a wide range of small volume realtime chemical and biochemical analyses. The integrated microfluidic label-free analysis platform uses an analysis cartridge consisting of Si-NW sensor arrays, for (bio)chemical detection and analysis, integrated with a small volume microfluidic flow-cell. The low power Si-NW (bio)chemical sensor cartridge has been developed for an automated molecular diagnostic system that is integrated in an ingestible pill. Sample delivery in the integrated analytical instrument is performed with an automated multi-sample injection system that eliminates erroneous sensor responses from sample switching due to flow rate fluctuations and provides precise sample volumes. The sample transport to the sensor surface is controlled to ensure that reaction-limited binding models can be used to estimate the biomolecular affinity. We describe the components of the integrated measurement system and its use for meaningful data acquisition for real-time biosensing applications. We demonstrate the efficacy of the integrated system for biochemical sensing with real-time DNA-PNA (peptide nucleic acid) duplex hybridization measurements together with an estimation of the binding affinity. Chemical sensing is performed by measuring sample $\mathrm{pH}$ under flow conditions of sequentially injected sample buffers.

\section{Experimental}

\section{Materials}

Si-NW sensors were fabricated from silicon-on-insulator (SOI) wafers (G4P-005-02, Soitec). Triangular Si-NWs have been fabricated using the previously reported method developed by our group that uses conventional microlithography, wet anisotropic etching ( $5 \%$ tetramethyl-ammonium-hydroxide) and size reduction, oxidation, and dry etching. ${ }^{13}$ The microfluidic chip is manufactured from poly-dimethylsiloxane (PDMS) cast on a silicon mold using a $10: 1$ pre-polymer (Sylgard 184) to curing agent (Dow Corning) mixture. Inlet and outlet holes were formed in the microfluidic flow-cell with a punch tool $(\phi 0.5 \mathrm{~mm}$ Unicore, Harris). All hydraulic connections between the regulated pressure source (MFCS-8C, Fluigent), sample reservoir tubes (T341-6TLST $2 \mathrm{~mL}$, Econo Lab), sample injection valve (Cheminert Nanovolume 10-port valve, Valco Instruments Co. Inc.) and microfluidic chip are made with $360 \mu \mathrm{m}$ outside diameter polyether-ether-ketone (PEEK) tubing (TPK10X, Valco Instruments Co. Inc.). Amine functional groups are conjugated to the gate-oxide sensor surface using a 3-amino-propyl-triethoxy silane (APTES) monolayer. The amine-functionalized surface was activated with a $5 \mathrm{mM}$ sulfo-succinimide-maleimide cyclohexane carboxylate cross-linker (SSMCC, Pierce-Thermo Scientific). A synthetic 15-mer thiolated PNA probe was used for all hybridization experiments with sequence Cys-TGT-ACA-TCACAA-CTA- $\mathrm{NH}_{2}$. The complementary $(M O)$ DNA sequence is $5^{\prime}$ TAG-TTG-TGA-TGT-ACA-3' (Eurogentec). The non-complementary (NC) DNA sequence is $5^{\prime}$-ATC-AAC-ACT-ACA-TGT-3' (Eurogentec). DNA-PNA duplex hybridization on the gate-oxide of $\mathrm{Si}^{-}$ NWs was performed in a $1 \mathrm{mM}$ phosphate buffer with $\mathrm{pH}$ 7.4. A fluorophore (Cy5) labeled PNA probe (Eurogentec) was used as a control to confirm conjugation of the PNA probe to the gateoxide sensor surface.

\section{Methods}

Integrated analysis platform. The integrated analysis system consists of an automated multi-sample delivery subsystem that can deliver precise sample volumes to the integrated microfluidic analysis cartridge using regulated pressure sources. Fig. 1 shows a schematic diagram of the integrated system. The electrical biasing and measurement equipment are connected to the Si-NW biosensors. The microfluidic analysis cartridge and samples are housed in a Faraday cage to eliminate external

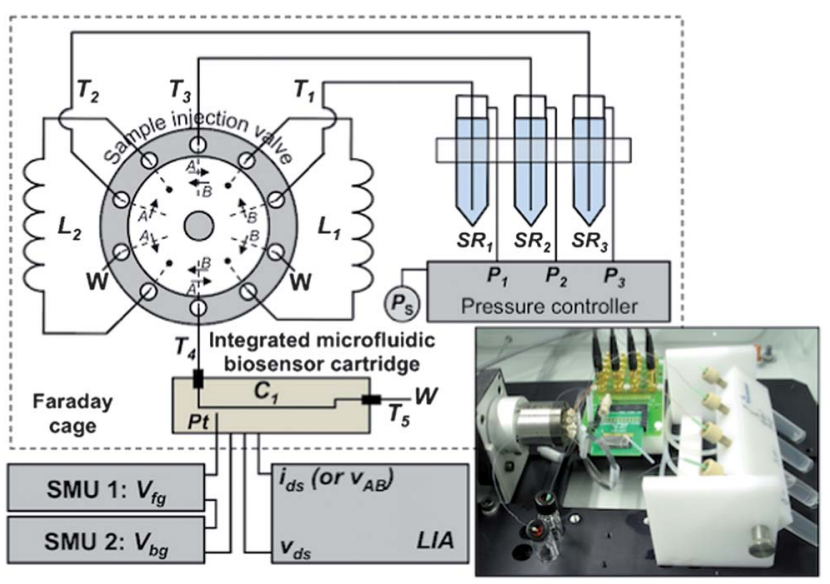

Fig. 1 Schematic diagram of the integrated label-free analysis system with automated multi-sample delivery to the microfluidic chip. PEEK tubing is used for all hydraulic connections $T_{1}-T_{5}$ and sample loops $L_{1}$ and $L_{2}$. $C_{1}$ is the microfluidic flow-cell channel. The pressures $P_{1}-P_{3}$ control the flow rate in tubes $T_{1}-T_{3}$, respectively. The dotted enclosure represents the Faraday cage. The electrical biasing and measurement equipment are connected to the Si-NW chip using coaxial cables and a common ground point on the custom-made circuit board. Inset: image of the analysis system. The sample injection valve has two switch positions $\mathrm{A}$ and $\mathrm{B}$. 
electrical and ambient light noise sources. The sample injection valve is used to selectively deliver different sample solutions from sample reservoirs $\mathrm{SR}_{1}, \mathrm{SR}_{2}$ and $\mathrm{SR}_{3}$, to the microfluidic flow-cell from pre-loaded sample loops, $L_{1}$ or $L_{2}$. The sample loops provide the capability to deliver a sample plug with volume from about $10 \mathrm{nl}$ (ref. 14) up to a larger volume that is dependent on the length of the sample loops. Since the Si-NW sensors are sensitive to surface potential changes, flow rate changes during sample injections can lead to significant and erroneous sensor responses due to the changes in the streaming potential at the gate-oxide surface induced by flowing electrolyte solutions. ${ }^{15}$ Low ionic strength samples are most problematic, e.g. we have found that the electrical response of the Si-NW sensors to varying flow rates of DI water and low ionic strength buffer solutions, e.g. $1 \mathrm{mM} \mathrm{NaCl}$ buffer solution ( $\mathrm{pH}$ 7.4), can be significant and often interfere with biomolecular hybridization sensor responses, as shown in the ESI. $\dagger$ The nanovolume sample injection valve, which has a small internal dead volume, and pressure driven sample transport provide stable and rapid sample switching when combined with a flow network that has balanced hydraulic resistances. The sample loop volume, sample flow rate, and experiment duration have been used to determine the length and inner diameter of the PEEK tubing, $T_{1}, T_{2}, T_{3}, T_{4}$ and $T_{5}$ and source pressures $P_{1}-P_{3}$, as shown in the ESI. $\dagger$ For sensing experiments, the flow network has been designed to provide a sample flow velocity range of $0.1 \mathrm{~mm} \mathrm{~s}^{-1}$ $\leq v_{\mathrm{s}} \leq 1 \mathrm{~mm} \mathrm{~s}^{-1}$ over an applied pressure range of $0.1 \mathrm{bar} \leq P_{\mathrm{i}}$ $\leq 1$ bar and variable experimental duration. For equilibrium association and dissociation constant estimation of hybridized biomolecular complexes, a flow speed $v_{\mathrm{s}} \sim 1 \mathrm{~mm} \mathrm{~s}^{-1}$ has been used, which is compatible with the reaction-limited transport of target molecules to the sensor surface.

Microfluidic flow-cell. Following surface cleaning of the Si-NW sensors, and subsequent modification with probe biomolecules as required, the Si-NW sensor chip is attached and wire bonded to a custom-made circuit board. The assembled Si-NW sensor chip is then aligned and clamped to the PDMS microfluidic chip, which is simple and non-destructive to the sensor surfaces and probe biomolecules on the sensor surfaces. Fig. 2a shows a schematic diagram of the integrated microfluidic biosensor analysis cartridge. The inlet and outlet holes of the PDMS microfluidic channel are connected to the sample injection valve with PEEK tubing. For experiments presented in this article the PDMS microfluidic channel is $1 \mathrm{~cm}$ in length and 360 $\mu \mathrm{m}$ in diameter. A platinum wire (267171, Sigma-Aldrich) pseudo-reference electrode is inserted directly into the microfluidic channel. Fig. 2b shows an example of an integrated microfluidic biosensor cartridge with inlet and outlet holes that are interfaced directly to the PEEK tubing. The analysis cartridge is mounted and wire bonded to a custom-made circuit board.

Si-NW sensors. The fabrication technology is capable of producing high-density Si-NW arrays with device diameters down to $10 \mathrm{~nm}$. The Si-NW sensors are boron doped $\left(N_{\mathrm{a}} \sim 10^{18} \mathrm{~cm}^{-3}\right)$ with heights between $100 \mathrm{~nm}$ and $140 \mathrm{~nm}$, measured with atomic force microscopy (AFM). Boron doping was done with conventional ion implantation and $\mathrm{N}_{2}$ anneal at $1000{ }^{\circ} \mathrm{C}$ for 30 minutes for
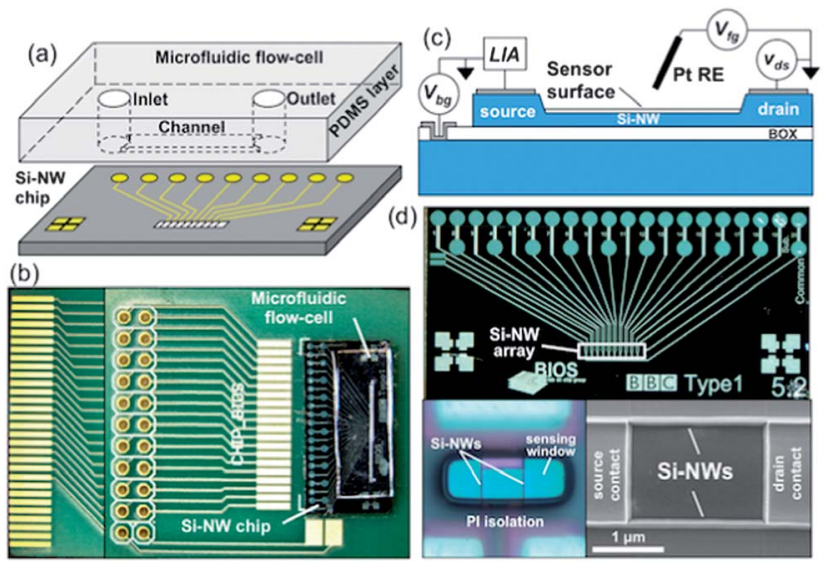

Fig. 2 Integrated microfluidic biosensor cartridge consisting of a Si-NW biosensor chip integrated with a microfluidic flow-cell. (a) Schematic of a Si-NW sensor chip and a PDMS microfluidic flow-cell. (b) Optical microscopy image of the implemented integrated microfluidic biosensor cartridge with the Si-NW biosensor chip attached and wire bonded to a custom circuit board, and a bonded microfluidic flow-cell. (c) Length cross-section schematic of a Si-NW biosensor with back-gate $V_{\mathrm{bg}}$, front-gate $V_{\mathrm{fg}}$ and drain-source $V_{\mathrm{ds}}$ bias voltages. Liquid gating of the Si-NW is controlled with a Pt reference electrode (RE) connected to $V_{\text {fg. }}$. A lock-in amplifier (LIA) measures the sensor current. (d) Microscopy images of fabricated Si-NWs. Upper: Si-NW sensor chip $(1 \mathrm{~cm} \times 2 \mathrm{~cm})$. Lower left: Si-NW sensor encapsulated with a PI layer and patterned sensing window. Lower right: high-resolution SEM image of a 2-wire Si-NW sensor.

dopant drive-in and activation. The aluminium electrical contacts have been annealed at $450{ }^{\circ} \mathrm{C}$ for 20 minutes in an $\mathrm{H}_{2} / \mathrm{N}_{2}$ forming gas to reduce interface effects and ensure ohmic and low metalsilicon contact resistance. The Si-NW chips were then encapsulated with a $2.5 \mu \mathrm{m}$ thick polyimide (PI) (annealed at $350^{\circ} \mathrm{C}$ for $1 \mathrm{~h}$ ) electrical isolation layer that has lithographically patterned sensing windows over the Si-NW sensor regions and electrical contact regions. A $15 \mathrm{~nm}$ thick silicon dioxide gate-oxide layer was used for $\mathrm{pH}$ measurements and a $2 \mathrm{~nm}$ thick gate-oxide layer was used for DNA hybridization experiments. Fig. 2c schematically shows the Si-NW length cross-section with all electrical bias voltages and electrical current measurement with a lock-in amplifier (LIA). Fig. $2 \mathrm{~d}$ shows an example of the Si-NW chip with an array of 2-wire Si-NW sensors (white highlighting) and a patterned sensing window in the PI isolation layer is shown in the lower left image of Fig. 2d. A high-resolution scanning electron microscopy (SEM) image that is representative of Si-NWs fabricated with this technology is shown in the lower right image of Fig. $2 \mathrm{~d}$.

Sensor surface preparation. Following encapsulation of the Si-NW sensor chip with the PI isolation layer, and prior to sensing experiments, the gate-oxide surfaces are cleaned with a UV-ozone treatment (UV/Ozone ProCleaner Plus, Bioforce Nanosciences) for 3 minutes. Gate-oxide surface cleaning with UV ozone before each measurement is essential for proper device operation. The electrical operation of the Si-NWs is optimal with short ozone treatment times; however, prolonged exposure to UV can degrade performance through the creation of surface states at the gate-oxide-silicon interface. ${ }^{16}$ Exposure continuously for more than 15 minutes resulted in non-functional devices, which is especially problematic for NWs as they have a very large surface-to-volume ratio and are extremely 
sensitive to interface properties. The device operation degradation, as a result of surface states, is observed by a continuous reduction of conductance to zero, which typically occurs within seconds. This kind of radiation damage can be reduced with low temperature annealing. ${ }^{17}$

pH sensor. The surface potential $\psi_{\mathrm{o}}$ of the Si-NW gate-oxide is dependent on the $\mathrm{pH}$ of the sample solution and is obtained through the titration of the gate-oxide in the presence of a supporting electrolyte. The Si-NW sensor surface is first cleaned with UV ozone, as previously described, and then the Si-NW sensor chip is attached and wire bonded to a custom circuit board and clamp-bonded with the PDMS microfluidic chip. The platinum reference electrode is inserted into the microfluidic flow channel. Prior to solution measurements, a $0.1 \mathrm{M} \mathrm{NaCl}$ supporting electrolyte with $0.01 \mathrm{M}$ universal buffer mixture (UBM: $0.01 \mathrm{M}$ citric acid, $0.01 \mathrm{M}$ phosphoric acid, and $0.02 \mathrm{M}$ boric acid) solution at $\mathrm{pH} 4$ was flowed from $\mathrm{SR}_{2}$ (Fig. 1) through the microchannel for 20 minutes to equilibrate the sensor surface. ${ }^{18}$ The $\mathrm{pH} 7$ and 10 solutions were realized by titrating the starting solution with a $1 \mathrm{M} \mathrm{NaOH}$ solution.

DNA hybridization. The surface potential $\psi_{\mathrm{o}}$ of the Si-NW gateoxide is dependent on the intrinsic electronic charge of biomolecules, such as DNA hybridized with complementary probes attached near the sensor surface. A sensor response is obtained by measuring the device conductance change, which is dependent on surface potential changes as target biomolecules accumulate on the gate-oxide surface in the presence of an aqueous buffer. Following cleaning of the gate-oxide surfaces with UV ozone, amine functionalized monolayers were silanized to the gate-oxide with APTES in 95\% ethanol and 5\% deionized water for 1 hour. The surface was then washed with ethanol and followed by a cross-linking heat treatment at $110{ }^{\circ} \mathrm{C}$ for 15 minutes (Fig. 3a). The surface is then activated with the SSMCC cross-linker in a $100 \mathrm{mM}$ triethanolamine buffer with $\mathrm{pH} 7.2$ for 1 hour (Fig. 3b). The surface is then incubated in $1 \mu \mathrm{M}$ thiolated PNA in the same buffer for 12 hours (Fig. 3c). We used a $60 \mu \mathrm{l}$ volume PDMS incubation well clamped to the Si-NW chip placed in a humidity chamber for all surface chemistry steps. The efficacy of the PNA probe attachment is demonstrated by comparing Fig. 3d and e. A negative control surface without a maleimide functional group was incubated with a PNA-Cy5 conjugate, and following a washing step we ideally expect that the Si-NW sensor regions are black, however, some fluorescence is detected due to non-specific PNA adsorption (Fig. 3d). The bright red regions are due to the silicon nitride layer covering the drain-source contacts. The silicon dioxide layer thickness in the sensor region is approximately $340 \mathrm{~nm}$ and quenching of the Cy5 fluorophore by the silicon is not possible in this case. The non-specific adsorption of PNA to glass is well known and we will return to this later in the article. A positive control surface containing an amine functional group following crosslinking activation and incubation with the PNA-Cy5 conjugate shows a uniform red color across the surface and confirms the PNA attachment to the gate-oxide surface (Fig. 3e). Subsequent studies of DNA-PNA hybridized duplexes attached to an oxide surface using radioactive labeling indicate surface densities $N_{\mathrm{d}} \sim 10^{12}$ molecules $\mathrm{cm}^{-2}$.



Fig. 3 PNA attachment to the Si-NW gate-oxide surface. (a-c) PNA attachment scheme. (d) Negative control incubated with a PNA-Cy5 conjugate. A single Si-NW sensor pair is shown. (e) Positive control incubated with PNA-Cy5. Three Si-NW sensor pairs are shown.

Electrical measurements. Si-NW FET sensors measure surface charge density $\sigma_{\mathrm{o}}$ on the gate-oxide sensor surface, from potential determining surface ions and/or the intrinsic electronic charge of hybridized biomolecular complexes attached to the sensor surface. The surface charge density induces a potential at the gate-oxide surface $\psi_{\mathrm{o}} \approx 2 k T q^{-1} \sinh ^{-1}\left(\sigma_{\mathrm{o}} / \beta\right)$, where $\beta=\left(8 \varepsilon_{\mathrm{w}} \varepsilon_{\mathrm{o}} k T c_{\mathrm{o}}\right)^{1 / 2}, \varepsilon_{\mathrm{w}}$ is the dielectric constant of the aqueous sample, $\varepsilon_{\mathrm{o}}$ is the permittivity of free space and $c_{\mathrm{o}}$ is the ionic strength of the background electrolyte. ${ }^{19}$ In practice, Si-NW FET sensors measure a change in surface potential $\Delta \psi_{\mathrm{o}}$ from a quiescent state due to chemical interactions at the gateoxide surface in the form of a sensor conductance change $\Delta G$ (i.e. $\Delta G=\Delta\left|i_{\mathrm{ds}}\right| / v_{\mathrm{ds}}$, where $\Delta\left|i_{\mathrm{ds}}\right|$ is the change in the NW current and $v_{\mathrm{ds}}$ is the ac voltage applied across the length of the $\mathrm{NW}$, as shown in Fig. 2c) in the silicon layer due to the electric field generated across the gate-oxide. The back-gate bias voltage $\left(V_{\mathrm{bg}}\right)$ controls the depletion region of the bottom side of the NW body, and the front-gate bias voltage $\left(V_{\mathrm{fg}}\right)$ controls the depletion behavior of the top sensor surfaces that are in contact with the solution using the reference electrode.

For all measurements presented in this article, the device operation was controlled with dc voltage sources $V_{\mathrm{fg}}$ and $V_{\mathrm{bg}}$ (2400 SMU, Keithley). The platinum wire reference electrode provides the front-gate bias in solution. The Si-NW is driven with an ac $(30 \mathrm{~Hz})$ modulation voltage $v_{\mathrm{ds}}$ and the ac device current is converted to a voltage with a transconductance amplifier (TA) and the magnitude and phase of the signal are measured with a lock-in amplifier (LIA) (SR 830, Stanford Research Systems). For single sensor measurements the internal TA of the LIA is used (Fig. 4a). The LIA uses quadrature synchronous demodulation, with an internal oscillator that is frequency matched to $v_{\mathrm{ds}}$, to retrieve the magnitude and phase 
(a)

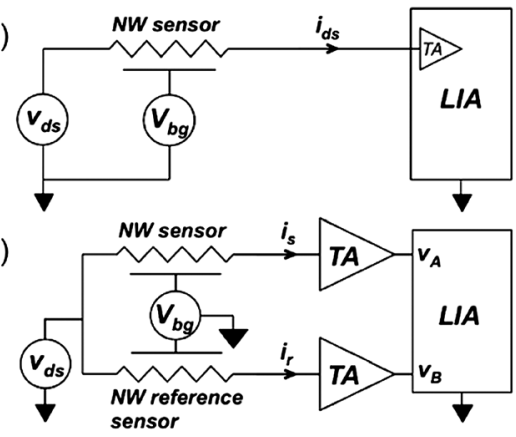

Fig. 4 Electrical measurement configurations. (a) Single-device measurement circuit. (b) Differential measurement circuit.

of $i_{\mathrm{ds}}$. We report the magnitude of the measured signal from the LIA, i.e. $\left|i_{\mathrm{ds}}\right|$. All biasing and measurement instruments are controlled using a custom-made Labview program.

pH and DNA hybridization sensor. Following cleaning of the gate-oxide sensor surface, and subsequent surface modification for biosensing applications, the integrated microfluidic sensor cartridge is placed in the integrated system. PEEK tubing attached to the inlet port was connected directly to the pressurized sample reservoir tubes $\left(\mathrm{SR}_{1}, \mathrm{SR}_{2}\right.$ and $\left.\mathrm{SR}_{3}\right)$, which are filled with $2 \mathrm{ml}$ of sample buffers with varying $\mathrm{pH}$ or DNA concentrations. The buffer solution was pumped through the system. For $\mathrm{pH}$ sensor experiments, the front-gate response curve $V_{\mathrm{fg}}-\left|i_{\mathrm{ds}}\right|$ was first recorded and analyzed in the initial solution ( $\mathrm{pH} 4$ ). The NW current $\left|i_{\mathrm{ds}}\right|$ was recorded for $\mathrm{pH} 7$ and 10 solutions, injected sequentially from $\mathrm{SR}_{2}$ and $\mathrm{SR}_{3}$, flowing through the microfluidic flow-cell. The measured $\mathrm{pH}$ sensitivity $\Delta \psi_{\mathrm{o}} / \Delta \mathrm{pH}$ was then calculated using the measured front-gate calibration curve (ref. 20). The hybridization buffer for DNA hybridization experiments is transported from $\mathrm{SR}_{1}$ until the surface was stabilized, i.e. about 20 minutes. The different DNA concentration samples were then injected and the corresponding $\left|i_{\mathrm{ds}}\right|$ was recorded for the duration of the experiment.

Differential measurements. A differential measurement configuration (Fig. $4 \mathrm{~b}$ ) provides the capability to cancel environmental sources of noise and interference, thus yielding measurements that better represent hybridization events compared to single sensor configurations. For differential measurements, two external TA (SIM918, Stanford Research Systems) are used to convert the currents from the reference and sensor devices into voltages $v_{\mathrm{A}}$ and $v_{\mathrm{B}}$, respectively. The LIA measurement is then performed in differential mode $\left(v_{A}-v_{B}\right)$.

\section{Results and discussion}

\section{Integrated Si-NW sensor operation and characterization}

Si-NW FET sensors measure surface potential changes $\Delta \psi_{\mathrm{o}}$ on the sensor surface that originate from changes in the surface charge density $\Delta \sigma_{\mathrm{o}}$ due to changes in surface ions and/or the intrinsic charge of attached biomolecular complexes, as previously described. The surface potential change $\Delta \psi_{\mathrm{o}}$ is determined from measurements of $\Delta G$ from homogeneously doped p-type depletion-mode Si-NWs. We recently reported that the conductance of depletion-mode Si-NWs can be operated in either the depletion or accumulation regions, thus the conductance is $G=q \mu_{\mathrm{b}} N_{\mathrm{a}} L^{-1} \xi+2 \eta \mu_{\mathrm{a}} W_{\mathrm{a}} L^{-1} Q_{\mathrm{a}}$, where $q$ is the electronic charge, $\mu_{\mathrm{b}}$ is the dopant concentration dependent bulk hole mobility, $N_{\mathrm{a}}$ is the boron impurity dopant concentration in the NW body, $L$ is the device length, $\xi$ is a gating function that is dependent on $\psi_{\mathrm{o}}$ (see ESI $\dagger$ ), $\mu_{\mathrm{a}}$ is the field dependent hole mobility in the accumulated layer, $W_{\mathrm{a}}$ is the total surface area of the accumulated surface and $Q_{\mathrm{a}}$ is the electronic charge in the accumulation layer. ${ }^{21}$ For all measurements presented in this article, the Si-NWs are operated in the depletion region where $Q_{\mathrm{a}}=0$ since $\left|V_{\mathrm{fg}}\right|<\left|V_{\mathrm{fb}}\right|$, where $V_{\mathrm{fb}}$ is the flatband voltage that represents all potential drops across the gate-oxide-silicon interface. It should be noted that the conductance associated with the metal/semiconductor drain and source contact regions has been carefully designed and characterized to have a negligible contribution to the total device conductance (see ESI + ). In summary, there are a few important points to highlight regarding the practical operation of Si-NW FET sensors: (i) the application of a dual gate biasing configuration with a reference electrode in the sample buffer ensures optimal and reproducible device behavior; (ii) the sensor surface should be well cleaned (e.g. short duration UV ozone) and the detrimental effects from the fixed oxide charges and surface states at the gate-oxide-silicon interface minimized through thermal annealing; and (iii) the contact resistance of the drain and source contacts should be minimized such that it represents a small fraction of the total quiescent sensor resistance.

The Si-NWs were characterized prior to experiments to assess the front-gate $V_{\mathrm{fg}}-\left|i_{\mathrm{ds}}\right|$ and $g_{\mathrm{m}}-\left|i_{\mathrm{ds}}\right|$ response curves, where $g_{\mathrm{m}}=\partial\left|i_{\mathrm{ds}}\right| / \partial V_{\mathrm{fg}}$ ( $v_{\mathrm{ds}}$ constant) is the sensor transconductance. Front-gate biasing affects the depletion region differently compared to back-gate biasing, as previously described, and for biosensor applications, the devices can be biased in the region with highest $g_{\mathrm{m}}$ and linear $\left|i_{\mathrm{ds}}\right|$ range while maintaining a low front-gate current $i_{\mathrm{fg}}<1 \mathrm{nA} .^{21,22}$ The back-gate bias $V_{\mathrm{bg}}$ is determined and fixed during the initial calibration. Fig. 5a shows a representative example of a $V_{\mathrm{fg}}-\left|i_{\mathrm{ds}}\right|$ response curve of a Si-NW where the front-gate voltage is scanned with a platinum reference electrode in a low ionic strength buffer ( $\mathrm{pH} 4)$ and $\left|i_{\mathrm{ds}}\right|$ recorded. The operation region transitions from depletion to accumulation as $V_{\mathrm{fg}}$ is scanned to larger negative voltages. The decrease in $g_{\mathrm{m}}$ for $V_{\mathrm{fg}}$ beyond the maximum is due to a decrease in field-dependent effective mobility $\mu_{\mathrm{a}}$ and charge screening. For all experiments, the Si-NW devices are biased with $V_{\mathrm{fg}}=-0.6 \mathrm{~V}$ and $V_{\mathrm{bg}}=-7.5 \mathrm{~V}$, unless specified otherwise.

pH sensor. Si-NW pH sensors measure the potential difference $\psi_{\mathrm{o}}$ across the electrical double layer at the interface between an electrolyte and the gate-oxide surface, which is related to the activity of the potential determining ions, which is described by the Nernst equation $\psi_{\mathrm{o}}=2.303 \mathrm{kTq}^{-1} \log \left[a_{\mathrm{H}^{+}} /{ }^{\circ} a_{\mathrm{H}^{+}}\right]$, where $a_{\mathrm{H}^{+}}$and ${ }^{\mathrm{o}} a_{\mathrm{H}^{+}}$are the ion activity of the solution and point of zero charge, respectively. Therefore, the maximum variation $\psi_{\text {o }}$ can take is $58 \mathrm{mV}$ per decade of ion activity at the standard temperature of $20^{\circ} \mathrm{C}$. The $\mathrm{H}^{+}$ion is primarily responsible for producing the surface potential on an oxide surface and 

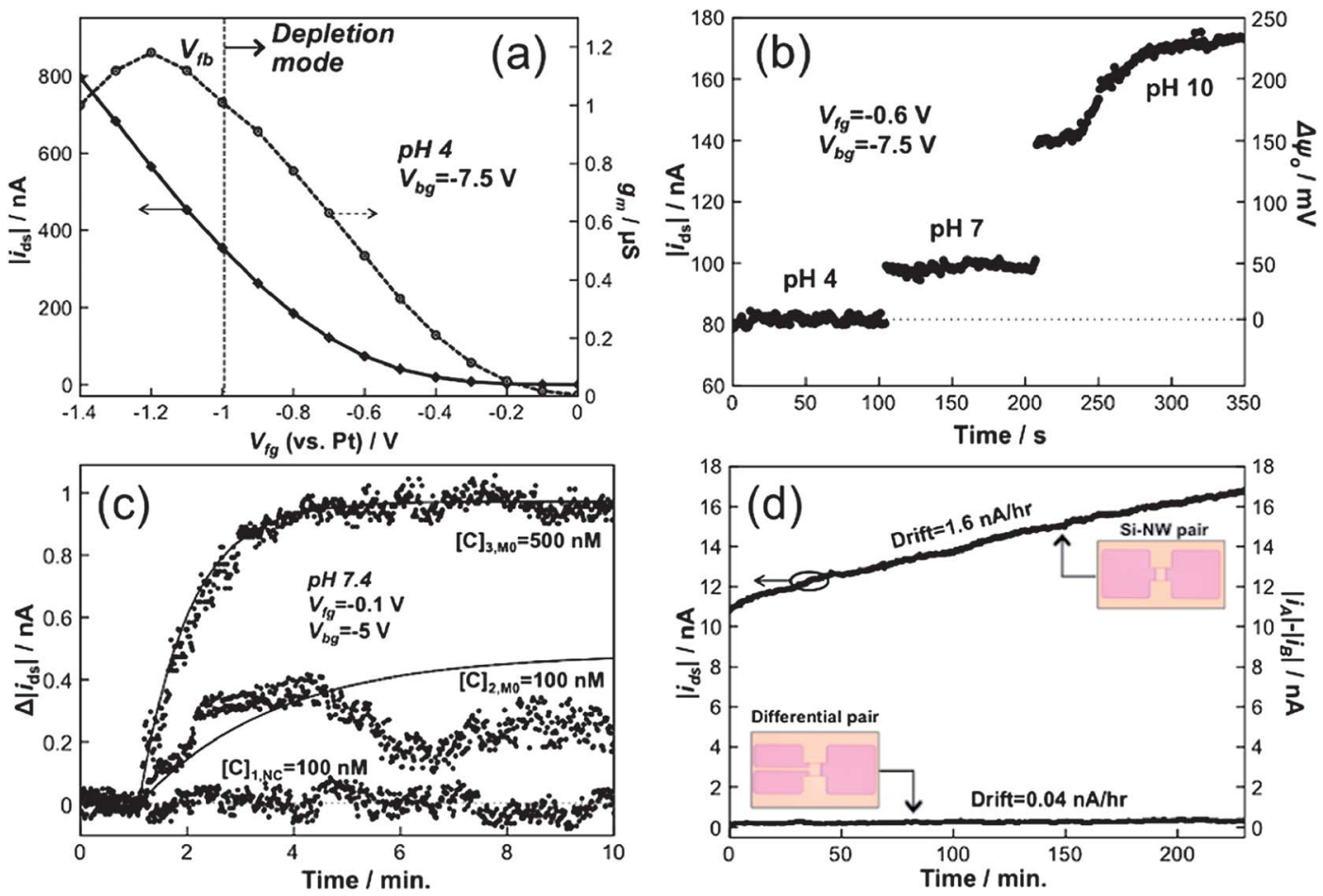

Fig. 5 Integrated biosensor analysis platform measurements. (a) Si-NW front-gate biasing characteristics in $\mathrm{pH} 4$ buffer solution with a platinum reference electrode. (b) $\mathrm{pH}$ measurements with flow speed $v_{\mathrm{s}} \approx 0.1 \mathrm{~mm} \mathrm{~s}^{-1}$. (c) DNA-PNA duplex hybridization detection with $[C]_{3, \mathrm{MO}}=500 \mathrm{nM},[C]_{2, \mathrm{MO}}=100 \mathrm{nM}$ and $[C]_{1, \mathrm{NC}}=100 \mathrm{nM}$ target concentrations and flow speed $v_{\mathrm{s}} \approx 0.6 \mathrm{~mm} \mathrm{~s}^{-1}$. (d) Differential measurement in buffer solution.

counter-ions present in solution are from the background electrolyte and can be neglected at low ionic concentrations. The experimental measurement of $\Delta \psi_{\mathrm{o}}$ is obtained through titration of the oxide in the presence of a supporting electrolyte by changing the $\mathrm{pH}$. The corresponding maximum $\mathrm{pH}$ sensitivity of a surface is, therefore, limited to $\Delta \psi_{\mathrm{o}} / \Delta \mathrm{pH}=$ $2.303 \mathrm{kTq}^{-1}$.

The $\mathrm{pH}$ measurements using the integrated microfluidic SiNW sensor platform were carried out using $v_{\mathrm{ds}}=100 \mathrm{mV}, V_{\mathrm{bg}}=$ $-7.5 \mathrm{~V}$ and UV ozone cleaned gate-oxide sensor surfaces. Firstly, a pH 4 buffer solution was transported through the microfluidic flow-cell for 20 minutes to establish a stable baseline signal, and followed by the injection of a buffer solution with $\mathrm{pH} 7$ for 2 minutes and finally the injection of a $\mathrm{pH} 10$ buffer for 3 minutes, all at a flow rate of $0.5 \mu \mathrm{min}^{-1}$, which corresponds to an average flow speed $v_{\mathrm{s}} \approx 0.1 \mathrm{~mm} \mathrm{~s}^{-1}$. For p-type depletion mode Si-NWs, increasing the solution $\mathrm{pH}$ results in an increasingly negative surface potential change, which results in a reduced depletion region of the depletion-mode Si-NW devices, and therefore, an increase in $\left|i_{\mathrm{ds}}\right|$. Fig. $5 \mathrm{~b}$ shows the $\mathrm{pH}$ response of the integrated Si-NW sensor system to different $\mathrm{pH}$ sample solutions. From Fig. 5a, the slope of the $V_{\mathrm{fg}}-\left|i_{\mathrm{ds}}\right|$ response curve is $\Delta\left|i_{\mathrm{ds}}\right| / \Delta V_{\mathrm{fg}} \approx-2.6 \mathrm{mV} \mathrm{nA}^{-1}$ (at $V_{\mathrm{fg}}=-0.6 \mathrm{~V}$ ) over the $\mathrm{pH}$ experiment range. The right-side ordinate of Fig. $5 \mathrm{~b}$ shows the estimated surface potential change as the solution is increased from $\mathrm{pH} 4$ to $\mathrm{pH} 10$. Note that the surface potential change is estimated from the initial experimental sample with $\mathrm{pH} 4$. The bare gate-oxide surfaces demonstrate the well-known nonlinearity for low $\mathrm{pH}$ values near the point of zero charge
$\left(\mathrm{pH}_{\mathrm{pzc}} \approx 2\right)$, which becomes linear for $\mathrm{pH} 5$ and above. ${ }^{18,21}$ The measured current shows a sharp increase followed by a gradual increase to the steady state response for an increase in $\mathrm{pH}$ from 7 to 10 , which is characteristic of the time dependent surface equilibration of the oxide surface. Over the range $7 \leq \mathrm{pH} \leq 10$ the $\mathrm{pH}$ sensitivity is $\Delta \psi_{\mathrm{o}} / \Delta \mathrm{pH} \approx 48 \mathrm{mV} \mathrm{pH}^{-1}$, which is consistent with measurements in previous experimental results using conventional laboratory sample volumes. $^{21}$ This measurement demonstrates that the integrated system can provide stable and well-controlled $\mathrm{pH}$ sensing with small sample volumes over the time scale of minutes. Other sensing surfaces, such as $\mathrm{Al}_{2} \mathrm{O}_{3}$ or $\mathrm{Ta}_{2} \mathrm{O}_{5}$, which are well known to provide near Nernstian sensitivity and linear $\mathrm{pH}$ responses, ${ }^{21,23}$ can be easily incorporated into the Si-NW sensing chip.

DNA hybridization. Binding of negatively charged DNA biomolecules near the Si-NW gate-oxide surface induces a change in the surface potential $\Delta \psi_{\mathrm{o}}$, which results in $\Delta G$ of the Si-NW, as previously discussed, provided that the intrinsic biomolecular charge is not screened due to ionic species in the electrical double layer at the gate-oxide surface; ${ }^{24}$ i.e. the Debye length $\left(\lambda_{\mathrm{D}}\right)$ (ref. 25) of the buffer solution at the gate-oxide surface should not be too small (i.e. $\lambda_{\mathrm{D}}>5 \mathrm{~nm}$ for vertically attached probes). Counter-ion screening, or counter-ion condensation, has also been reported for DNA molecules where the negative charges on the phosphate backbone are screened by cations in the electrolyte, thus reducing the intrinsic DNA charge by up to $80 \%$ for high ionic strength solutions. ${ }^{26}$ Therefore, low ionic strength $(<100 \mathrm{mM})$ hybridization buffers are preferred for the highest sensitivity detection. However, low 
ionic strength hybridization buffers are not suitable for high efficiency DNA-DNA duplex formation. For this purpose we use synthetic PNA probes, mimics of oligonucleotides with a charge neutral peptide backbone, which are reported to have low equilibrium dissociation constants to complementary DNA in low ionic strength buffers that have a relatively large Debye length; the DNA-PNA duplex formation is less dependent on the ionic strength of the hybridization buffer compared to DNA-DNA duplex formation. ${ }^{27-29}$

We present real-time DNA-PNA duplex hybridization measurements of complementary MO DNA target molecules to PNA probe molecules covalently conjugated to the silicon oxide gate-oxide surface of 2-wire Si-NW sensors (Fig. 3b) in a low ionic strength buffer. The measurements have been performed with sample flow speeds high enough to ensure reaction-limited transport of the target DNA to the sensor surface and we use the first-order Langmuir binding model to assess the equilibrium dissociation constant $K_{\mathrm{D}}$ of the DNA-PNA duplex hybridization process. The equilibrium dissociation constant is defined as $K_{\mathrm{D}}=$ $k_{\mathrm{a}} / k_{\mathrm{d}}$, where $k_{\mathrm{a}}$ is the association rate constant that represents the speed of the second-order probe-target interaction and $k_{\mathrm{d}}$ is the dissociation rate constant that represents the speed of the firstorder breakdown of the probe-target complex. Thermodynamics dictates the target-probe hybridization process and a small $K_{\mathrm{D}}$ value indicates a high affinity of the probe for the target, which generally lies in the $10^{-15} M<K_{\mathrm{D}}<10^{-6} \mathrm{M}$ range. ${ }^{30}$

From mass action kinetics, the time dependent surface density formation of a single type of probe-target complex on the surface can be monitored in real-time with the response function $R(t)=R_{\mathrm{o}}\left(1-\exp \left[-\left(k_{\mathrm{a}}[C]+k_{\mathrm{d}}\right) t\right]\right)$, where $R_{\mathrm{O}}=$ $\left(k_{\mathrm{a}} R_{\max }[C]\right) /\left(k_{\mathrm{a}}[C]+k_{\mathrm{d}}\right), R_{\max }$ is the maximum sensor signal response for a given probe density $N_{\mathrm{p}}$ and $[C]_{\mathrm{i}}$ is the concentration of the target analyte. The time dependent response function, which is derived by solving a rate equation that includes the association and dissociation of target molecules with surface probe molecules as a function of time, is commonly referred to as the Langmuir equation. The real-time response measured from the Si-NW sensors is drain-source current, thus $R(t) \equiv\left|i_{\mathrm{ds}}\right|(t)$ for fixed biases $V_{\mathrm{fg}}, V_{\mathrm{bg}}$ and $v_{\mathrm{ds}}$, and target concentration $[C]_{\mathrm{i}}$ for each measurement. Fig. 5c shows measured real-time current responses for injections of $M O$ DNA with $[C]_{2, \mathrm{M} 0}=100 \mathrm{nM}$ and $[C]_{3, \mathrm{M} 0}=500 \mathrm{nM}$ concentrations in $1 \mathrm{mM}$ phosphate buffer $\left(\lambda_{\mathrm{D}} \approx\right.$ $10 \mathrm{~nm}$ ) to complementary PNA probe molecules conjugated to the gate-oxide surface with a sample flow speed of $v_{\mathrm{s}} \approx 0.6 \mathrm{~mm} \mathrm{~s}^{-1}$. The sensor response to the injection of NC DNA with $[C]_{1, \mathrm{NC}}=$ $100 \mathrm{nM}$ is also shown in Fig. 5c, which is the baseline noise from the measurement. The $500 \mathrm{nM}$ concentration $M O$ DNA produces $\Delta\left|i_{\mathrm{ds}}\right| \approx 1 \mathrm{nA}$ and $100 \mathrm{nM} M O$ DNA produces $\Delta\left|i_{\mathrm{ds}}\right| \approx 0.4 \mathrm{nA}$, as shown in Fig. $5 \mathrm{c}$. The estimated limit of detection is $50 \mathrm{nM}$, using a 3-sigma cutoff of the measured NC100 nM data noise, for the experimental conditions used in this article and can be reduced, which will be discussed later in this section. The increase in conductance with an increase of negatively charged molecules on the gate-oxide sensor surface of the p-type depletion mode sensor is expected from the sensor characterization (Fig. 5a). We have measured the density of DNA-PNA duplexes formed on oxide surfaces in equilibrium, using a ${ }^{32} \mathrm{P}$ radioactive assay (data not shown), and found that a saturated surface contains about $N_{\mathrm{d}}$ $\sim 10^{12}$ duplexes $\mathrm{cm}^{-2}$, which corresponds to a lower limit $\Delta \psi_{\mathrm{o}} \sim$ $10 \mathrm{mV}$ (ref. 31) using an oxide charge density $\sigma_{\mathrm{ox}} \approx 0.2 \mathrm{C} \mathrm{m}^{-2}$ for a buffer with $\mathrm{pH} 7 .^{21}$ The estimated surface potential changes correspond to larger conductance changes compared to the measured conductance changes and we are currently working to optimize the PNA probe sensor surface.

The association and dissociation rate constants, $k_{\mathrm{a}}$ and $k_{\mathrm{d}}$, are extracted by fitting the measured current responses to the kinetics response function using all of the experimental concentrations. The solid lines in Fig. $5 \mathrm{c}$ represent the model fit to the measured data with $R_{\max } \approx 1 \mathrm{nA}, k_{\mathrm{a}} \approx 3 \times 10^{4} \mathrm{M}^{-1} \mathrm{~s}^{-1}$ and $k_{\mathrm{d}} \approx 4 \times 10^{-3} \mathrm{~s}^{-1}$, which gives $K_{\mathrm{D}}=140 \mathrm{nM}$. The $R_{\max }$ value is consistent with $[C]_{3, \mathrm{M} 0}>K_{\mathrm{D}}$, where all PNA binding sites are assumed to be occupied. The extracted $K_{\mathrm{D}}$ is much larger than dissociation constants extracted from measurements of the DNA-PNA duplex hybridization..$^{28,29,32}$ A dissociation constant of $K_{\mathrm{D}} \approx 350 \mathrm{nM}$ for a DNA-PNA duplex hybridization at $35^{\circ} \mathrm{C}$ has been reported..$^{33}$ The discrepancy between the measurements can be due to differences in the PNA attachment scheme. ${ }^{29}$ Extracted $K_{\mathrm{D}}$ values of DNA-DNA duplex hybridization measured using Si-NW sensors in a $165 \mathrm{mM}$ ionic strength buffer are similar to our measurements. ${ }^{12}$

We have found in subsequent studies that PNA molecules non-specifically adsorb to many types of surfaces. Although it is well known that PNA molecules non-specifically adsorb to glass surfaces (Eurogentec), they also adsorb non-specifically to silicon nitride, PDMS and polyimide surfaces with similar attachment densities, and are difficult to remove in most cases (data not shown). The non-specific adsorption of PNA on the polyimide surface is problematic and can negatively affect the estimation of the dissociation rate $k_{\mathrm{d}}$ and the limit of detection to target depletion. Following the kinetics and transport analysis that describe the interaction of target analytes with probe molecules on surfaces, ${ }^{34-36}$ we can further assess the validity of our measurements. From the channel dimensions and sample flow rate, the transport of target DNA to the sensing surface is reaction-limited since the Damkohler number $\mathrm{Da} \approx 2 \times 10^{-3}$, thus verifying the use of the single complex formation response function. From Fig. 5c, the time constant $\tau_{\mathrm{r}}=\left(k_{\mathrm{a}}[C]_{\mathrm{i}}+k_{\mathrm{d}}\right)^{-1}$ of the $100 \mathrm{nM}$ response $\tau_{\mathrm{r}} \approx 2$ minutes and the $500 \mathrm{nM}$ response has $\tau_{\mathrm{r}} \approx 1$ minute, which is much smaller than the time constants measured in previous DNA-PNA duplex formation studies where $\tau_{\mathrm{r}} \sim 10$ minutes have been reported. ${ }^{28,29}$ Fast DNA-PNA and DNA-DNA duplex hybridization times have been reported in other Si-NW sensor studies. ${ }^{12,37}$ More work is required to understand these differences and to optimize the PNA probe density and minimize the non-specific adsorption of PNA to surfaces that interface with the target DNA.

Differential sensing. Sensor drift is the systematic increase or decrease of the sensor response as a function of measurement time. It is well known that the output response of ion sensitive field-effect transistor sensors, the microscale predecessors to Si-NW FET sensors, suffer from drift due to ion migration at the gate-oxide interface. ${ }^{\mathbf{8} 38}$ A typical response of the 2-wire Si-NW sensor (Fig. 2d) with a silicon dioxide surface, measured with the single device measurement configuration 
shown in Fig. 4, in a $1 \mathrm{mM} \mathrm{NaCl}$ buffer solution over a period of about 4 hours, is shown in the upper curve in Fig. $5 d$. The drift rate of $1.6 \mathrm{nA} \mathrm{h} \mathrm{h}^{-1}$ is significant considering the quiescent current $\left|i_{\mathrm{ds}}\right| \approx 10 \mathrm{nA}$. Note that the left ordinate scale represents the measured current from the single device measurement configuration. A differential measurement configuration can be used to improve the output response of the Si-NW sensors. Fig. 4b shows an example of the differential measurement configuration where the current is divided into reference and sensor currents, $i_{\mathrm{s}}$ and $i_{\mathrm{r}}$, respectively, using the split-source Si-NW device, shown in the ESI. $†$ The currents are converted to voltages $v_{\mathrm{A}}$ and $v_{\mathrm{B}}$ with external TAs and the LIA output in differential measurement mode represents their difference, or null value $\left(v_{\mathrm{A}}-v_{\mathrm{B}}\right)$. A typical differential response is shown in the lower curve in Fig. $5 \mathrm{~d}$, where the drift rate has been significantly reduced to $0.04 \mathrm{nA} \mathrm{h}^{-1}$, which is reduced by a factor of $\sim 30 \times$. Note that the right ordinate scale represents the difference of the measured currents from the differential measurement configuration. The quiescent output signal is ideally nulled to zero (in this case the offset current is $0.1 \mathrm{nA}$ due to device mismatch), and therefore, changes in the sensor signal $\Delta i_{\mathrm{s}}$ can be detected with a higher sensitivity as the detection range of the instrument can be reduced. If the sensor and reference devices are each replaced by $N$-parallel connected Si-NW devices then the detection response will be ideally amplified by a factor of $N \times$. For example, when the single sensor and reference devices are replaced by a parallel array of $\mathrm{N}$-SiNWs the ideal nulled output signal is $i_{\mathrm{s}}-i_{\mathrm{r}}=N \Delta i$, where $\Delta i$ is the current change from a single NW sensor induced from a surface potential change $\Delta \psi_{\mathrm{o}}$. The LIA measurement instrument readout is a voltage difference $v_{\mathrm{A}}-v_{\mathrm{B}} \approx-N v_{\mathrm{ds}}\left(R_{\mathrm{f}}\right)$ $\left.R_{\mathrm{NW}}\right)\left(\Delta R / R_{\mathrm{NW}}\right)$, where $R_{\mathrm{f}}$ is the gain of the TA, $R_{\mathrm{NW}}$ is the quiescent resistance of the NW and $\Delta R$ is the change in the NW resistance induced $\Delta \psi_{\mathrm{o}}$.

\section{Conclusions}

We have presented an integrated microfluidic label-free analytical platform that can be used for small volume chemical analysis, such as $\mathrm{pH}$ or ion sensing, as well as diagnostics for the specific measurement of biomolecules and for the kinetics estimation of biomolecular hybridization. We have integrated a scalable silicon nanowire sensor chip with a microfluidic flowcell into a simple disposable cartridge format that is mounted into the all-electrical readout instrument. An automated multisample injection system was presented that provides fast and reliable sample switching without spurious flow rate fluctuations that can produce erroneous sensor signals. Measurements of $\mathrm{pH}$ from different samples have been presented with sensitivities consistent with a silicon dioxide sensor surface. Realtime DNA-PNA duplex hybridization measurements have been presented with different sample concentrations in a low ionic strength buffer, and the equilibrium dissociation constant $K_{\mathrm{D}}$ $\approx 140 \mathrm{nM}$ has been extracted from the experimental data using the first order Langmuir binding model. Finally, a differential sensor configuration has been presented and a $30 \times$ reduction in sensor drift has been shown. The integrated microfluidic label-free analysis platform with a measurement cartridge based on an electronic Si-NW biosensor array, integrated with a simple microfluidic flow-cell, has been demonstrated by measuring different sample types. The Si-NW (bio)chemical sensor cartridge has been developed for an automated molecular diagnostic system integrated in an ingestible pill, and is a promising platform for low cost (bio)chemical diagnostics and drug screening.

\section{Acknowledgements}

This work was supported by the Nanopill project funded by a private cancer research foundation in the Netherlands and the Beyond Moore NanoNextNL Program funded by the Government of the Netherlands. The authors thank Johan Bomer for helpful comments on device processing and measurements, Hans de Boer for assistance with the measurement setup and chip holder, the MESA+ Nanolab staff, Mark Smithers for assistance with SEM imaging, and Rico Keim for TEM sample preparation and imaging.

\section{Notes and references}

1 P. Bertone and M. Snyder, FEBS J., 2005, 272, 5400.

2 N. Ramachandran, D. N. Larson, P. R. Stark, E. Hainsworth and J. LaBaer, FEBS J., 2005, 272, 5412.

3 W. Gu, Trends in Bio/Pharmaceutical Industry, 2009, 5, 43.

4 J. Comley, Drug Discovery World, 2008, Fall 28.

5 J. Homola, S. S. Yee and G. Gauglitz, Sens. Actuators, B, 1999, $\mathbf{5 4}, 3$.

6 G. Krishnamoorthy, E. T. Carlen, J. G. Bomer, A. van den Berg and R. B. M. Schasfoort, Lab Chip, 2010, 10, 986.

7 P. M. Levine, P. Gong, R. Levicky and K. L. Shepard, Biosens. Bioelectron., 2009, 24, 1995.

8 P. Bergveld, Sens. Actuators, 1981, 1, 17.

9 J. Fritz, E. B. Cooper, S. Gaudet, P. K. Sorger and S. R. Manialis, Proc. Natl. Acad. Sci. U. S. A., 2002, 99, 14142.

10 E. T. Carlen and A. van den Berg, Lab Chip, 2007, 7, 19.

11 Y. Cui, Q. Q. Wei, H. K. Park and C. M. Lieber, Science, 2001, 293, 1289.

12 Y. Bunimovich, Y. S. Shin, W.-S. Yeo, M. Amori, G. Kwong and J. R. Heath, J. Am. Chem. Soc., 2006, 128, 16323.

13 S. Chen, J. G. Bomer, W. G. van der Wiel, E. T. Carlen and A. van den Berg, ACS Nano, 2009, 3, 3485.

14 Assuming a PEEK tubing sample loop with a diameter of 25 $\mu \mathrm{m}$ and a length of $2 \mathrm{~cm}$.

15 D. R. Kim, C. H. Lee and X. L. Zheng, Nano Lett., 2009, 9, 1984.

16 E. H. Snow, A. S. Grove and D. J. Fitzgerald, Proc. IEEE, 1967, 55, 1168.

17 B. E. Deal, J. Electrochem. Soc., 1974, 121, C198.

18 L. Bousse, N. F. de Rooij and P. Bergveld, IEEE Trans. Electron Devices, 1983, 30, 1263.

19 J. Israelachvili, Intermolecular and Surface Forces, Academic Press, 2nd edn, London, 1992.

20 Measurements were performed at $20{ }^{\circ} \mathrm{C}$. A gel pH electrode (PHC10101 electrode, Hach Lange) and a multimeter 
(HQ40D multi, Hach Lange) were used for $\mathrm{pH}$ measurements and calibration. The $\mathrm{pH}$-meter was calibrated at $\mathrm{pH} 4.0$ and pH 10.0 before each measurement.

21 S. Chen, J. G. Bomer, E. T. Carlen and A. van den Berg, Nano Lett., 2011, 11, 2334.

22 H. D. Tong, S. Chen, W. G. van der Wiel, E. T. Carlen and A. van den Berg, Nano Lett., 2009, 9, 1015.

23 O. Knopfmacher, A. Tarasov, W. Y. Fu, M. Wipf, B. Niesen, M. Calame and C. Schonenberger, Nano Lett., 2010, 10, 2268.

24 J. Janata, in Proc. of the 2nd Int. Meeting on Chemical Sensors, ed. J. L. Aucouturier et al., Bordeaux, France, 1986, p. 25.

25 Debye length is defined as $\lambda_{\mathrm{D}}=\left(\varepsilon_{\mathrm{w}} \varepsilon_{\mathrm{o}} k T / 2 z^{2} q^{2} c_{\mathrm{o}}\right)^{1 / 2}$, where $z$ is the valence ionic charge and $c_{\mathrm{o}}$ is the ionic concentration. For example, $\lambda_{\mathrm{D}} \approx 10 \mathrm{~nm}$ for $c_{\mathrm{o}}=1 \mathrm{mM}$ for a monovalent ion electrolyte solution.

26 G. S. Manning, Q. Rev. Biophys., 1978, 11, 179.

27 M. Egholm, O. Buchardt, L. Christensen, C. Behrens, S. M. Freier, D. A. Driver, R. H. Berg, S. K. Kim, B. Norden and P. E. Nielsen, Nature, 1993, 365, 566.
28 J. Liu, L. Tiefenauer, S. Tian, P. E. Nielsen and W. Knoll, Anal. Chem., 2006, 78, 470.

29 H. Park, A. Germini, A. Sforza, R. Corradini, R. Marchelli and W. Knoll, Biointerphases, 2007, 2, 80.

30 D. A. Lauffenburger and J. J. Linderman, Receptors: Models for Binding, Trafficking, and Signaling, Oxford University Press, New York, 1993.

31 The estimated surface potential $\Delta \psi_{\mathrm{o}} \approx\left(2 k T_{\mathrm{q}}{ }^{-1}\right)\left(\sinh ^{-1}\right.$. $\left.\left(\left(\sigma_{\text {DNA }}-\sigma_{\text {ox }}\right) / \beta\right)-\sinh ^{-1}\left(\sigma_{\text {ox }} / \beta\right)\right)$, where $\varepsilon_{\mathrm{w}}=80$ and $c_{\mathrm{o}}=1$ mM. The charge density of a saturated 15-mer DNA-PNA surface is estimated with $\sigma_{\mathrm{DNA}} \approx N_{\mathrm{d}} \times 15 \times q \times 10^{4} \mathrm{C} \mathrm{m}^{-2}$. 32 F. Yu, D. Yao and W. Knoll, Nucleic Acids Res., 2004, 32, e75. 33 K. Jensen, H. Orum, P. E. Nielsen and B. Norden, Biochemistry, 1997, 36, 5072.

34 S. Sjölander and C. Urbanicky, Anal. Chem., 1991, 63, 2338. 35 M. Zimmerman, E. Delamarche, M. Wolf and P. Hunziker, Biomed. Microdevices, 2005, 7, 99.

36 T. M. Squires, R. J. Messinger and S. R. Manalis, Nat. Biotechnol., 2008, 26, 417.

37 J. Hahm and C. M. Lieber, Nano Lett., 2004, 4, 51.

38 R. G. Kelly, Electrochim. Acta, 1977, 22, 1. 